\title{
Regularity and Passivity for Jump Rules in Linear Switched Systems
}

\author{
Giuliano Costantini $i^{\sharp}$, Stephan Trenn $\diamond$, Francesco Vasca ${ }^{\natural}$
}

\begin{abstract}
A wide class of linear switched systems (LSS) can be represented by a sequence of modes each one described by a set of differential algebraic equations (DAEs). LSS can exhibit discontinuities in the state evolution, also called jumps, when the state at the end of a mode is not consistent with the DAEs of the successive mode. Then the problem of defining a proper state jump rule arises when an inconsistent initial condition is given. Regularity and passivity conditions provide two conceptually different jump maps respectively. In this paper, after proving some preliminary result on the jump analysis within the regularity framework, it is shown the equivalence of regularitybased and passivity-based jump rules. A switched capacitor electrical circuit is used to numerically confirm the theoretical result.
\end{abstract}

Index Terms-Switched systems, Passivity-based jump rule, Regularity, Wong sequences, Inconsistent initial conditions.

\section{INTRODUCTION}

When modeling electrical circuits which exhibit sudden structural changes (e.g. component faults or opening/closing of ideal switches) an interesting question is how the state variables jump in order to satisfy the new algebraic constraints. A good overview of the different jump-rules is given in [1]. A quite general modeling framework for linear switched systems are switched differential algebraic equations (switched DAEs) [2] given as follows

$$
E_{\sigma(t)} \dot{\xi}(t)=A_{\sigma(t)} \xi(t)
$$

where $\sigma: \mathbb{R} \rightarrow\{1, \ldots, P\}$ is the switching signal and $E_{p}, A_{p} \in \mathbb{R}^{n \times n}$ determine the dynamics (and algebraic constraints) in each mode $p \in\{1, \ldots, P\}$ of the system.

A global solution is constructed by concatenating these single modes whose solutions can be obtained separately as solutions of

$$
\begin{aligned}
& E_{p} \dot{\xi}(t)=A_{p} \xi(t) \quad t \in[0, \infty) \\
& \xi\left(0^{-}\right)=\xi^{0} \in \mathbb{R}^{n} .
\end{aligned}
$$

A classic solution exists only when the initial condition (2b) fulfills the algebraic constraints which arise in the current mode.

When modeling electrical circuits with ideal switches only, a more economic way of modeling is via a standard statespace system with constant matrices and virtual inputs and outputs, which are complementary and encode the mode of the position of the switches [1], i.e. open circuit or short circuit. In particular, in each switching configuration some

\footnotetext{
$(\sharp)$ University of Kaiserslautern, Germany, email: giuliano. costantini@ymail.com

$(\diamond)$ Department of Mathematics, University of Kaiserslautern, 67663 Kaiserslautern, Germany, email: trenn@mathematik.uni-kl.de

( ) Department of Engineering, University of Sannio, Piazza Roma 21, 82100 Benevento, Italy, email: vasca@unisannio.it
}

of the "outputs" are set to zero leading to a DAE. In this framework the following principles are used to derive a jump rule in response to an inconsistent initial value:

1) Minimization of the energy.

2) Conservation of the charge/flux.

3) The Laplace transform approach.

In [1] it was shown that under a certain passivity assumption these three approaches lead to equivalent jump rules, which we will call passivity-based jump rule in the following.

Under a certain regularity assumption one can prove [2] that for any switching signal and any initial condition the switched DAE (1) (or equivalently (2)) has a unique solution (but within a distributional solution framework). In particular, the regularity assumption implies unique jumps at the switching times. The jump rule based on regularity was not studied in [1] while passivity was not considered in [2]; but, of course, electrical circuits which are passive and regular play an important role and the question arises whether the passivity-based jump rule is equivalent to the regularity-based jump rule. The answer is yes and is our main result (Theorem 6) we would like to present.

The paper is structured as follows. In Section II we present our system class of linear switched systems and how each mode can be rewritten as a DAE. The passivity-based jump rule is introduced in Section III and afterwards the regularitybased jump rule is introduced. Our main result is given in Section V. We illustrate the theoretical results with a basic switched capacitor electrical circuit example.

We use the following notation: For $x \in \mathbb{R}^{n}, u \in \mathbb{R}^{m}$ we let $\operatorname{col}(x, u) \in \mathbb{R}^{n+m}$ be the vector which results in stacking $x$ over $u$. For a matrix $M \in \mathbb{R}^{p \times q}$ and a subspace $\mathcal{S} \subseteq \mathbb{R}^{q}$ the subspace $M \mathcal{S} \subseteq \mathbb{R}^{p}$ denotes the image space of $\mathcal{S}$ under $M$, i.e. $M \mathcal{S}:=\left\{M s \in \mathbb{R}^{p} \mid s \in \mathcal{S}\right\}$; for $\mathcal{S} \subseteq \mathbb{R}^{p}$ the subspace $M^{-1}(\mathcal{S}) \subseteq \mathbb{R}^{q}$ denotes the preimage of $\mathcal{S}$ under $M$, i.e. $M^{-1}(\mathcal{S}):=\left\{x \in \mathbb{R}^{q} \mid M x \in \mathcal{S}\right\}$. For $v \in \mathbb{R}^{n}$ and a set of integers $\mathcal{N} \subseteq\{1,2, \ldots, n\}$, with $v_{\mathcal{N}}$ we refer to the vector obtained from $v$ by considering only the components in the set $\mathcal{N}$. Similarly, if $M \in \mathbb{R}^{n_{1} \times n_{2}}$ is a matrix, with $M_{\mathcal{N}_{1} \mathcal{N}_{2}}$ we refer to the matrix obtained from $M$ by considering only the rows in the set $\mathcal{N}_{1} \subseteq\left\{1,2, \ldots, n_{1}\right\}$ and the columns in the set $\mathcal{N}_{2} \subseteq\left\{1,2, \ldots, n_{2}\right\}$. The symbol $\bullet$ stands for the whole set of rows or columns.

\section{Linear Switched Systems AND DAEs}

A linear switched system (LSS) within a linear complementarity framework can be described as follows [1], [3]

$$
\begin{aligned}
\dot{x}(t) & =\bar{A} x(t)+\bar{B} z(t) \\
w(t) & =\bar{C} x(t)+\bar{D} z(t)
\end{aligned}
$$


together with the complementarity condition

$$
z_{i}(t) w_{i}(t)=0 \quad \forall i=1,2, \ldots, q \forall t \in \mathbb{R}
$$

where $\bar{A}, \bar{B}, \bar{C}, \bar{D}$ are matrices of suitable dimensions, $x(t) \in \mathbb{R}^{n}$ is called semi-state and the vectors $z(t) \in \mathbb{R}^{q}$ and $w(t) \in \mathbb{R}^{q}$ are ideal switch variables satisfying (4), i.e. at each time instant either the component $z_{i}$ or the corresponding component $w_{i}$ must be zero.

Such description is not to be confused with the switched linear system model as in [4] given by $\dot{x}=A_{\sigma} x$; in particular, the latter does not contain any algebraic constraints, hence the problem of inconsistent initial values is not present.

The solution of an LSS can be obtained by cascading the solutions of the models corresponding to the different modes. In that sense one can restrict the state jump analysis occurrence at the time instant when a generic mode is activated. Then without loss of generality in what follows the state jump at the initial time instant is considered and the indication of the subscript ' $p$ ' denoting the specific mode is omitted.

In order to select a mode of the system it is necessary to define a switch configuration through the subset $\pi \subseteq$ $\{1,2, \ldots, q\}$. Say $m$ is the size of the index set $\pi$. When $\pi$ is active the complementarity condition (4) becomes

$$
\begin{aligned}
w_{i}=0 & \text { if } i \in \pi \\
z_{i}=0 & \text { if } i \in \pi^{c},
\end{aligned}
$$

where $\pi^{c}=\{1,2, \ldots, q\} \backslash \pi$. By combining (3) and (5) we obtain for each switch configuration $\pi$ a simplified structure:

$$
\begin{aligned}
\dot{x} & =\bar{A} x+\bar{B} \bar{\bullet}_{\bullet} z_{\pi} \\
0 & =\bar{C}_{\pi \bullet} x+\bar{D}_{\pi \pi} z_{\pi} \\
z_{\pi^{c}} & =0 \\
w_{\pi^{c}} & =\bar{C}_{\pi^{c} \bullet} x .
\end{aligned}
$$

The model (6a)-(6b) can be simply rearranged into

$$
\begin{aligned}
\dot{x} & =K x+L u \\
0 & =M x+N u
\end{aligned}
$$

where $u=z_{\pi}, K=\bar{A}, L=\bar{B}_{\bullet \pi}, M=\bar{C}_{\pi \bullet}$, and $N=\bar{D}_{\pi \pi}$.

The model (7) can be rewritten in the form of a DAE. Let us define the state vector as $\operatorname{col}(x, u)$. Then (7) can be written as the following DAE

$$
E\left(\begin{array}{l}
\dot{x} \\
\dot{u}
\end{array}\right)=A\left(\begin{array}{l}
x \\
u
\end{array}\right)
$$

where $E, A \in \mathbb{R}^{(n+m) \times(n+m)}$ are square matrices given by

$$
E=\left[\begin{array}{ll}
I & 0 \\
0 & 0
\end{array}\right], \quad A=\left[\begin{array}{ll}
K & L \\
M & N
\end{array}\right]
$$

where $I$ is the identity matrix of order $n$. Note that the special structure of the matrix pair $(E, A)$ as in (9) is no restriction of generality as any matrix pair can be brought into this form via suitable coordinate transformations. However, in order to apply the passivity based analysis the special structure of (7) is necessary.

\section{StATE JUMPS}

In order to define a solution for (7), or equivalently for (8)(9), one must fix an initial condition, say $\operatorname{col}\left(x_{0}, u_{0}\right)$. It can be proved that for any initial condition there exists a solution of (8) in the distributional framework [2, Thm 6.5.1] provided the matrix pair $(E, A)$ is regular (see Section IV). The occurrence of a state jump can be formalized by means of the consistency concept.

Definition 1: An initial condition $\operatorname{col}\left(x_{0}, u_{0}\right)$ is called consistent for the DAE (8) if, and only if, there exists an absolutely continuous solution $\operatorname{col}(x(\cdot), u(\cdot))$ of (8) such that $\operatorname{col}(x(0), u(0))=\operatorname{col}\left(x_{0}, u_{0}\right)$. Otherwise the initial condition is called inconsistent.

Definition 2: The consistency space $\mathfrak{C}_{(E, A)}$ is the set of all vectors $\operatorname{col}\left(x_{0}, u_{0}\right)$ which represent a consistent initial condition for a given DAE.

\section{A. Projection Matrix}

Switching between different DAEs each given by (8) in general leads to inconsistent initial values because the consistency space need not to coincide. In order to obtain a solution the state must clearly jump to a consistent initial value first. In order to correctly define this state re-initialization a jump rule must be chosen which unambiguously maps an inconsistent initial value into a consistent one. Since we are in a linear framework, this jump rule must be linear and also should map consistent initial values on themselves, i.e. the jump rule is given by a projection matrix $\Pi \in$ $\mathbb{R}^{(n+m) \times(n+m)}$ :

$$
\left(\begin{array}{l}
x_{0}^{+} \\
u_{0}^{+}
\end{array}\right)=\Pi\left(\begin{array}{l}
x_{0}^{-} \\
u_{0}^{-}
\end{array}\right)
$$

Definition 3: We define the jump space $\mathfrak{J}_{\Pi}$ as the set of all vectors which represent a valid re-initialization for the jump rule taken into account, i.e.

$\mathfrak{J}_{\pi}:=\left\{\begin{array}{l|l}\Delta \in \mathbb{R}^{n+m} & \exists\left(\begin{array}{l}x_{0}^{-} \\ u_{0}^{-}\end{array}\right): \Pi\left(\begin{array}{l}x_{0}^{-} \\ u_{0}^{-}\end{array}\right)=\left(\begin{array}{l}x_{0}^{-} \\ u_{0}^{-}\end{array}\right)-\Delta\end{array}\right\}$
According to (10), since $\Pi$ is an idempotent matrix, it results

$$
\begin{aligned}
\mathfrak{C}_{(E, A)} & =\operatorname{im} \Pi \\
\mathfrak{J}_{\Pi} & =\operatorname{ker} \Pi
\end{aligned}
$$

with $\mathfrak{C}_{(E, A)} \oplus \mathfrak{J}_{\Pi}=\mathbb{R}^{n+m}$. Therefore, for a given projection matrix $\Pi$, any initial condition $\operatorname{col}\left(x_{0}^{-}, u_{0}^{-}\right) \in \mathbb{R}^{n+m}$ can be uniquely decomposed as follows

$$
\left(\begin{array}{l}
x_{0}^{-} \\
u_{0}^{-}
\end{array}\right)=\left(\begin{array}{l}
x_{0}^{+} \\
u_{0}^{+}
\end{array}\right)+\left(\begin{array}{c}
\Delta_{x} \\
\Delta_{u}
\end{array}\right)
$$

with $\operatorname{col}\left(x_{0}^{+}, u_{0}^{+}\right) \in \mathfrak{C}_{(E, A)}$ and $\operatorname{col}\left(\Delta_{x}, \Delta_{u}\right) \in \mathfrak{J}_{\Pi}$.

\section{B. Passivity-based Jump Rule}

A common re-initialization strategy [1] is based on the passivity-based jump rule. In order to apply such a rule the passivity concept must be introduced, which can be characterized as follows. 
Definition 4: [1, Prop IV.3] A quadruple $(\bar{A}, \bar{B}, \bar{C}, \bar{D})$ is passive if, and only if, the following linear matrix inequality

$$
\left[\begin{array}{cc}
\bar{A}^{\top} Q+Q \bar{A} & Q \bar{B}-\bar{C}^{\top} \\
\bar{B}^{\top} Q-\bar{C} & -\left(\bar{D}+\bar{D}^{\top}\right)
\end{array}\right] \leq 0 .
$$

has a positive semi-definite solution $Q=Q^{\top} \geq 0$. We call $(\bar{A}, \bar{B}, \bar{C}, \bar{D})$ stritly passive if (13) has a positive definite solution $Q=Q^{\top}>0$.

Assume that the quadruple $(\bar{A}, \bar{B}, \bar{C}, \bar{D})$ associated to the LSS (3) is strictly passive with a positive definite solution $Q>0$ of (13) and consider the system (7) resulting for a generic mode. It can be shown that the passivity-based jump rule for the semi-state $x$ is independent of $u$ and can be obtained by solving the following quadratic program with respect to $x_{0}^{+}[1, \mathrm{Thm}$ VI.1]:

$$
\begin{array}{ll}
\text { minimize } & \frac{1}{2}\left(x_{0}^{+}-x_{0}^{-}\right)^{\top} Q\left(x_{0}^{+}-x_{0}^{-}\right) \\
\text {subject to } & M x_{0}^{+} \in \operatorname{im} N .
\end{array}
$$

In particular, (14) has a unique solution which can be written as

$$
x_{0}^{+}=\Pi_{\text {pas }}^{x} x_{0}^{-}
$$

with

$$
\Pi_{\mathrm{pas}}^{x}=I-Q^{-1} M^{T} P^{T}\left(P M Q^{-1} M^{T} P^{T}\right)^{\dagger} P M
$$

where $\dagger$ refers to the pseudoinverse matrix and $P$ is a matrix such that $\operatorname{ker} P=\operatorname{im} N$.

Moreover, under the strict passivity condition, it is also proved in [1] that

$$
\begin{aligned}
& \operatorname{im} \Pi_{\text {pas }}^{x}=M^{-1}(\operatorname{im} N) \\
& \operatorname{ker} \Pi_{\text {pas }}^{x}=L \operatorname{ker} N
\end{aligned}
$$

in particular, $M^{-1}(\mathrm{im} N)$ is the consistency space for the semi-state $x$ and

$$
M^{-1}(\operatorname{im} N) \oplus L \operatorname{ker} N=\mathbb{R}^{n} .
$$

\section{Regularity AND Wong SEQUences}

An alternative way for defining a jump rule is based on the DAE representation (8) and on the concept of regularity.

Definition 5: A matrix pair $(E, A)$ is regular if $\operatorname{det}(s E-$ $A)$ is not the zero polynomial.

The regularity property allows to obtain an explicit solution formula for (7) by employing the quasi-Weierstrass form as described below.

Theorem 1 ([5]): Given a regular matrix pair $(E, A)$ there exist invertible matrices $S, T$ such that $(E, A)$ is transformed into a quasi-Weierstrass form

$$
(S E T, S A T)=\left(\left[\begin{array}{ll}
I & 0 \\
0 & Z
\end{array}\right],\left[\begin{array}{ll}
J & 0 \\
0 & I
\end{array}\right]\right)
$$

where $J$ is some matrix and $Z$ is a nilpotent matrix.

In view of [6] we call (19) quasi-Weierstrass form because we do not assume that $J$ and $Z$ are in Jordan canonical form.

A convenient method to find the transformation matrices $S, T$ is to employ the so called Wong sequences:
Definition 6 ([7]): Consider a matrix pair $(E, A)$, then the following subspace sequences are called Wong sequences:

$$
\begin{aligned}
\mathcal{V}_{i+1} & =A^{-1}\left(E \mathcal{V}_{i}\right) \\
\mathcal{W}_{i+1} & =E^{-1}\left(A \mathcal{W}_{i}\right)
\end{aligned}
$$

with $\mathcal{V}_{0}=\mathbb{R}^{n+m}, \mathcal{W}_{0}=\{0\}$.

Theorem 2: [6, Prep 2.4] If the matrix pair $(E, A)$ is regular then the Wong sequences get stationary after finitely many steps. Denote $\mathcal{V}^{*}$ and $\mathcal{W}^{*}$ the limits, and choose full rank matrices $V, W$ such that $\mathcal{V}^{*}=\operatorname{im} V$ and $\mathcal{W}^{*}=\operatorname{im} W$ then

$$
\begin{aligned}
T & =[V, W] \\
S & =[E V, A W]^{-1}
\end{aligned}
$$

yield the quasi-Weierstrass form (19).

It is easily seen that with the help of the quasi-Weierstrass form a unique jump rule based on the regularity assumption follows. In fact, in [2, Thm 6.5.1] it is proved that the consistency projector is given by

$$
\Pi_{\text {reg }}=T\left[\begin{array}{ll}
I & 0 \\
0 & 0
\end{array}\right] T^{-1}
$$

with the matrix $T$ given by (21a). Note that this projection maps onto $\mathcal{V}^{*}$ along $\mathcal{W}^{*}$, thus it holds

$$
\begin{aligned}
\mathfrak{C}_{(E, A)} & =\operatorname{im} \Pi_{\text {reg }}=\mathcal{V}^{*}, \\
\mathfrak{J}_{\Pi_{\text {reg }}} & =\operatorname{ker} \Pi_{\text {reg }}=\mathcal{W}^{*} .
\end{aligned}
$$

Since the matrix $E$ is assumed to have the special structure (9), in what follows some original preliminary results are obtained.

Firstly it can be proved that, similarly to the passivitybased jump rule, the vector $u_{0}$ does not influence reinitialization of the semi-state $x$.

Theorem 3: Consider the DAE (8) with the matrix $E$ given by (9) and assume the matrix pair $(E, A)$ is regular. By using the Wong sequences define the spaces $\mathcal{V}^{*}=\operatorname{im} V$ and $\mathcal{W}^{*}=\operatorname{im} W$ and the regularity projector (22). Then the following condition holds

$$
\Pi_{\text {reg }}\left(\begin{array}{l}
x_{0} \\
u_{0}
\end{array}\right)=\Pi_{\text {reg }}\left(\begin{array}{c}
x_{0} \\
0
\end{array}\right) .
$$

for all $x_{0} \in \mathbb{R}^{n}$ and $u_{0} \in \mathbb{R}^{m}$.

Proof: First notice that

$$
\{0\}=\mathcal{W}_{0} \subsetneq \mathcal{W}_{1} \subsetneq \cdots \subsetneq \mathcal{W}_{i}=\mathcal{W}_{i+1}=\mathcal{W}^{*} .
$$

Moreover

$$
\mathcal{W}_{1}=\left[\begin{array}{ll}
I & 0 \\
0 & 0
\end{array}\right]^{-1}\left(\left[\begin{array}{cc}
K & L \\
M & N
\end{array}\right] \mathcal{W}_{0}\right)=\operatorname{ker}\left[\begin{array}{ll}
I & 0 \\
0 & 0
\end{array}\right] \subseteq \mathcal{W}^{*}=\operatorname{ker} \Pi_{\mathrm{reg}} .
$$

Then

$$
\left(\begin{array}{l}
0 \\
u
\end{array}\right) \in \operatorname{ker}\left[\begin{array}{ll}
I & 0 \\
0 & 0
\end{array}\right] \subseteq \operatorname{ker} \Pi_{\mathrm{reg}}
$$

which with simple algebraic manipulations implies (24):

$$
\Pi_{\mathrm{reg}}\left(\begin{array}{l}
x \\
u
\end{array}\right)=\Pi_{\mathrm{reg}}\left[\left(\begin{array}{l}
x \\
0
\end{array}\right)+\left(\begin{array}{l}
0 \\
u
\end{array}\right)\right]=\Pi_{\mathrm{reg}}\left(\begin{array}{l}
x \\
0
\end{array}\right) .
$$


Since we are only interested in the semi-state $x$, in what follows we seek a restriction of the jump map. First we define the restriction of the spaces $\mathcal{V}^{*}$ and $\mathcal{W}^{*}$ relative to the variable $x$.

Theorem 4: Consider the DAE (8) with the matrix $E$ given by (9) and assume the matrix pair $(E, A)$ is regular. Let $\mathcal{V}^{*}$ and $\mathcal{W}^{*}$ be the spaces which terminate the Wong sequences. Define the projector

$$
\Pi_{x}=\left[\begin{array}{ll}
I & 0
\end{array}\right] .
$$

Then the spaces $\mathcal{V}_{x}^{*}=\Pi_{x} \mathcal{V}^{*}$ and $\mathcal{W}_{x}^{*}=\Pi_{x} \mathcal{W}^{*}$ fulfil

$$
\mathcal{V}_{x}^{*} \oplus \mathcal{W}_{x}^{*}=\mathbb{R}^{n}
$$

Proof: In the first part of the proof it is demonstrated that

$$
\mathcal{V}_{x}^{*} \cap \mathcal{W}_{x}^{*}=\{0\}
$$

Let $\bar{x} \in \mathcal{V}_{x}^{*} \cap \mathcal{W}_{x}^{*}$, then $\exists v \in \mathcal{V}^{*}, w \in \mathcal{W}^{*}$ such that

$$
\bar{x}=\Pi_{x} v \wedge \quad \bar{x}=\Pi_{x} w .
$$

Then it follows

$$
\begin{aligned}
\Pi_{x} v & =\Pi_{x} w \\
v-w & \in \operatorname{ker} \Pi_{x}=\operatorname{ker}\left[\begin{array}{ll}
I & 0 \\
0 & 0
\end{array}\right] \subseteq \mathcal{W}^{*} .
\end{aligned}
$$

Therefore since $w \in \mathcal{W}^{*}$ it is also true that $v \in \mathcal{W}^{*}$. Finally since $v \in \mathcal{V}^{*} \cap \mathcal{W}^{*}=\{0\}$ we obtain

$$
\bar{x}=\Pi_{x} v=0 .
$$

Choose $x \in \mathbb{R}^{n}$ and consider the vector $\operatorname{col}(x, 0) \in \mathbb{R}^{n+m}$, then there must exist a unique pair $v \in \mathcal{V}^{*}$ and $w \in \mathcal{W}^{*}$ such that

$$
\left(\begin{array}{l}
x \\
0
\end{array}\right)=v+w .
$$

It follows $x=\Pi_{x} v+\Pi_{x} w \in \mathcal{V}_{x}^{*}+\mathcal{W}_{x}^{*}$ and thus the claim.

Thus, in analogy of (12), any inconsistent initial value $x_{0}^{-}$ can be decomposed as follows

$$
x_{0}^{-}=x_{0}^{+}+\Delta_{x}
$$

with unique $x_{0}^{+} \in \mathcal{V}_{x}^{*}$ and $\Delta_{x} \in \mathcal{W}_{x}^{*}$. In particular, the jump-rule is given by a projector with image $\mathcal{V}_{x}^{*}$ and kernel $\mathcal{W}_{x}^{*}$, i.e. the regularity-based projector is given by choosing any two full rank matrices $V_{x}, W_{x}$ such that $\mathcal{V}_{x}^{*}=\operatorname{im} V_{x}$, $\mathcal{W}_{x}^{*}=\operatorname{im} W_{x}$ and

$$
\Pi_{\mathrm{reg}}^{x}=\left[V_{x}, W_{x}\right]\left[\begin{array}{cc}
I & 0 \\
0 & 0
\end{array}\right]\left[V_{x}, W_{x}\right]^{-1} .
$$

The following lemma shows that the regularity-based projector for the semi-state $x$ can also be calculated directly with the help of the consistency projector $\Pi_{\text {reg }}$ and the canonical projector $\Pi_{x}=[I, 0]$.

Lemma 1: Consider the DAE (8) with the matrix $E$ given by (9) and assume the matrix pair $(E, A)$ is regular. Say $\Pi_{\text {reg }}$ the corresponding consistency projector. Then

$$
\Pi_{\mathrm{reg}}^{x}=\Pi_{x} \Pi_{\mathrm{reg}} \Pi_{x}^{T} .
$$

TABLE I

CONSISTENCY AND JUMP SPACES

\begin{tabular}{c|c|c} 
& Regularity & Passivity \\
\hline Consistency space & $\mathcal{V}_{x}^{*}$ & $M^{-1} \mathrm{im} N$ \\
\hline Jump space & $\mathcal{W}_{x}^{*}$ & $L$ ker $N$
\end{tabular}

Relevant spaces for the re-initialization of the semi-state $x$.

The (surprisingly technical) proof is carried out in the Appendix.

The spaces $\mathcal{V}_{x}^{*}$ and $\mathcal{W}_{x}^{*}$ can also be obtained as the result of subspace iterations as the following result shows.

Theorem 5: Consider the DAE (8) with the matrix $E$ given by (9) and assume the matrix pair $(E, A)$ is regular. Then the following algorithm provides the space $\mathcal{V}_{x}^{*}$ and $\mathcal{W}_{x}^{*}$ after finitely many steps.

$$
\begin{aligned}
& \mathcal{V}_{x}^{i}=\left\{x \in \mathbb{R}^{n} \mid \exists u \in \mathbb{R}^{m}, \begin{array}{l}
K x+L u \in \mathcal{V}_{x}^{i-1} \\
M x+N u=0
\end{array}\right\} \\
& \mathcal{W}_{x}^{i}=\left\{x \in \mathbb{R}^{n} \mid \begin{array}{ll}
\bar{x} \in \mathcal{W}_{x}^{i-1} & \begin{array}{l}
x=K \bar{x}+L \bar{u} \\
\bar{u} \in \mathbb{R}^{m}
\end{array} \\
0=M \bar{x}+N \bar{u}
\end{array}\right\}
\end{aligned}
$$

with $\mathcal{V}_{x}^{0}=\mathbb{R}^{n}$ and $\mathcal{W}_{x}^{0}=\{0\}$

The proof is carried out in the Appendix.

\section{Equivalence Result}

In this section we prove the main result of the paper, i.e. the equivalence between regularity- and passivity-based jump rules. Therefore, we first assume that both regularity and strict passivity are satisfied by the system under study. Table I compares the fundamental spaces related to the $x$-restriction for both the aforementioned theories.

Theorem 6 (Equivalence between jump rules): Consider the DAE (8)-(9) and assume the matrix pair $(E, A)$ is regular and $(K, L, M, N)$ is strictly passive. Let the regularity-based jump projector $\Pi_{\text {reg }}^{x}$ be given as in Lemma 1 and let the the passivity-based jump-projector $\Pi_{\mathrm{pas}}^{x}$ be given by (16), then

$$
\Pi_{\text {reg }}^{x}=\Pi_{\text {pas }}^{x} .
$$

Proof: The first crucial observation is that we have two expressions for the consistency space for the semi-state $x$. One is given by the canonical projection $\mathcal{V}_{x}^{*}$ of the limit of the first Wong sequence $\mathcal{V}^{*}$ and the second one as the image of the passivity-jump projector, i.e.

$$
\mathcal{V}_{x}^{*}=M^{-1} \operatorname{im} N .
$$

A more careful analysis of the formula for $\mathcal{V}_{x}^{1}$ reveals

$$
\begin{aligned}
\mathcal{V}_{x}^{1} & =\left\{x \in \mathbb{R}^{n} \mid \exists u \in \mathbb{R}^{m}, M x+N u=0\right\} \\
& =\left\{x \in \mathbb{R}^{n} \mid M x \in \operatorname{im} N\right\} \\
& =M^{-1} \operatorname{im~} N=\mathcal{V}_{x}^{*} .
\end{aligned}
$$

Hence we can conclude that the $\mathcal{V}_{x}^{i}$-sequence ends after just one step. 


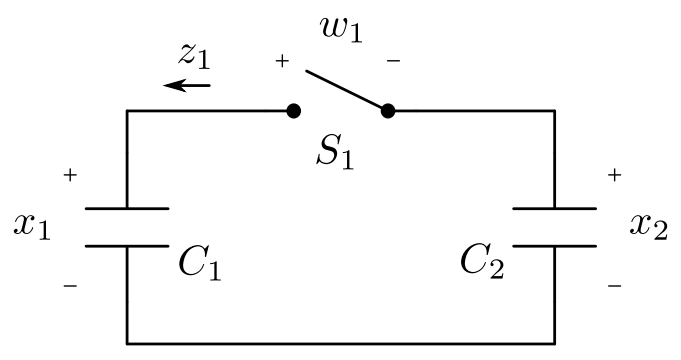

Fig. 1. Two capacitors connected in parallel by a switch.

Similarly, the calculation of $\mathcal{W}_{x}^{1}$ reveals

$$
\mathcal{W}_{x}^{1}=\left\{\begin{array}{l|l}
x \in \mathbb{R}^{n} \mid \bar{u} \in \mathbb{R}^{m} \begin{array}{l}
x=L \bar{u} \\
0=N \bar{u}
\end{array}
\end{array}\right\}=L \operatorname{ker} N .
$$

To prove the equality of the two jump-rules it remains to be shown that $\mathcal{W}_{x}^{*}=\mathcal{W}_{x}^{1}$.

From (18) it follows that

$$
\mathbb{R}^{n}=M^{-1}(\operatorname{im} N) \oplus L \operatorname{ker} N=\mathcal{V}_{x}^{*} \oplus \mathcal{W}_{x}^{1} .
$$

Invoking (29) this implies

$$
\operatorname{dim} \mathcal{W}_{x}^{1}=\operatorname{dim} \mathcal{W}_{x}^{*}
$$

and together with $\mathcal{W}_{x}^{1} \subseteq \mathcal{W}_{x}^{*}$ we can conclude $L$ ker $N=$ $\mathcal{W}_{x}^{1}=\mathcal{W}_{x}^{*}$.

\section{EXAMPLE}

In this section we deploy a canonical switched system, consisting of an electrical network with two capacitors connected together via an ideal switch (Fig. 1).

We first evaluate the projection maps $\Pi_{\text {reg }}^{x}$ and $\Pi_{\text {pas }}^{x}$ and then we compare them in order to show numerically the equivalence of the jump rules.

A straightforward analysis of the network lead us to

$$
\begin{aligned}
\left(\begin{array}{l}
\dot{x}_{1} \\
\dot{x}_{2}
\end{array}\right) & =\left[\begin{array}{ll}
0 & 0 \\
0 & 0
\end{array}\right]\left(\begin{array}{l}
x_{1} \\
x_{2}
\end{array}\right)+\left[\begin{array}{c}
\frac{1}{C_{1}} \\
-\frac{1}{C_{2}}
\end{array}\right] z_{1} \\
w_{1} & =\left[\begin{array}{ll}
1 & -1
\end{array}\right]\left(\begin{array}{l}
x_{1} \\
x_{2}
\end{array}\right)+[0] z_{1}
\end{aligned}
$$

with either $w_{1}=0$ or $z_{1}=0$.

The system has two different modes depending on the state of the switch $S_{1}$. When it is open the network current $z_{1}$ is forced to be null, vice versa when it is closed the voltage $w_{1}$ is zero.

Taking this into account, we consider the following scenario. Initially the switch is open and therefore the voltages across the capacitors remain constant to a certain value $x_{0}=\operatorname{col}\left(x_{01}, x_{02}\right)$. At the time $t=t_{0}$ the switch closes and the semi-state variables might receive a jump towards a value that is consistent for the new DAE.

The DAEs (8) which describe the system when the switch is closed are given by (40) with $w_{1}=0$ and $u=z_{1}$.

The consistency and jump spaces are

$$
\begin{aligned}
M^{-1} \operatorname{im} N & =\operatorname{ker} M=\operatorname{span}\left\{\left(\begin{array}{l}
1 \\
1
\end{array}\right)\right\} \\
L \operatorname{ker} N & =\operatorname{im} L=\operatorname{span}\left\{\left(\begin{array}{c}
1 / C_{1} \\
-1 / C_{2}
\end{array}\right)\right\} .
\end{aligned}
$$

The consistency projector relative to the $x$ variable can be easily derived

$$
\begin{aligned}
\Pi_{\mathrm{reg}}^{x} & =\left[\begin{array}{cc}
1 & 1 / C_{1} \\
1 & -1 / C_{2}
\end{array}\right]\left[\begin{array}{ll}
1 & 0 \\
0 & 0
\end{array}\right]\left[\begin{array}{cc}
1 & 1 / C_{1} \\
1 & -1 / C_{2}
\end{array}\right]^{-1} \\
& =\left[\begin{array}{cc}
\frac{C_{1}}{C_{1}+C_{2}} & \frac{C_{2}}{C_{1}+C_{2}} \\
\frac{C_{1}}{C_{1}+C_{2}} & \frac{C_{2}}{C_{1}+C_{2}}
\end{array}\right]
\end{aligned}
$$

In order to evaluate the passivity-based projector we need to solve the LMI problem in (13). To this end, Matlab LMI toolbox comes in handy by assigning numerical values to the network parameters. We chose: $C_{1}=1 \mu F$ and $C_{2}=2 \mu F$.

We find then a solution matrix

$$
Q=\left[\begin{array}{ll}
0.006051 & 0.012101 \\
0.012101 & 0.024203
\end{array}\right]
$$

and choosing a matrix $P=[1]$ we can finally evaluate the projector in (15)

$$
\Pi_{\mathrm{pas}}^{x}=\left[\begin{array}{ll}
0.3333 & 0.6667 \\
0.3333 & 0.6667
\end{array}\right] .
$$

A straightforward substitution of the numerical values in (41) leads to the same matrix of (42).

\section{CONCLUSIONS}

State jumps is an interesting phenomenon which occurs in linear switched systems due to the presence of modedependent algebraic constraints. The practical interest of this problem is demonstrated by the wide literature dedicated to the analysis of state jumps in electrical circuits. Different rules can be defined to re-initialize the state at a value which is consistent with the differential algebraic equations of the active mode. In this paper we have shown that the regularity based jump rule derived purely mathematical coincides with a jump rule based on physical considerations (energy minimization).

\section{APPENDIX}

\section{A. Proof Lemma 1}

Define $\Pi^{*}=\Pi_{x} \Pi_{\text {reg }} \Pi_{x}^{T}$.

1. $\Pi^{*} x=x \Leftrightarrow x \in \mathcal{V}_{x}^{*}$

Consider $x \in \mathcal{V}_{x}^{*}$, it results

$$
\Pi^{*} x=\Pi_{x} \Pi_{\mathrm{reg}} \Pi_{x}^{T} x=\Pi_{x} \Pi_{\mathrm{reg}}\left(\begin{array}{l}
x \\
0
\end{array}\right)
$$

and using Theorem 3

$$
\Pi_{x} \Pi_{\text {reg }}\left(\begin{array}{l}
x \\
0
\end{array}\right)=\Pi_{x} \Pi_{\text {reg }}\left(\begin{array}{l}
x \\
u
\end{array}\right) \quad \forall u \in \mathbb{R}^{m} .
$$

Choose now $\bar{u}: \operatorname{col}(x, \bar{u}) \in \mathcal{V}^{*}$, it follows

$$
\Pi^{*} x=\Pi_{x} \Pi_{\mathrm{reg}}\left(\frac{x}{\bar{u}}\right)=\Pi_{x}\left(\frac{x}{\bar{u}}\right)=x
$$


2. $\operatorname{im} \Pi^{*}=\mathcal{V}_{x}^{*}$.

$$
\text { im } \begin{aligned}
\Pi^{*} & =\operatorname{im} \Pi_{x} \Pi_{\mathrm{reg}} \Pi_{x}^{T} \\
& =\left\{\Pi_{x} \Pi_{\mathrm{reg}} \Pi_{x}^{T} x \mid x \in \mathbb{R}^{n}\right\} \\
& =\left\{\Pi_{x} \Pi_{\mathrm{reg}}\left(\begin{array}{c}
x \\
0
\end{array}\right) \mid x \in \mathbb{R}^{n}\right\} \\
& =\left\{\Pi_{x} \Pi_{\mathrm{reg}}\left(\begin{array}{c}
x \\
u
\end{array}\right) \mid\left(\begin{array}{c}
x \\
u
\end{array}\right) \in \mathbb{R}^{n+m}\right\} \\
& =\left\{\Pi_{x}\left(\begin{array}{c}
\bar{x} \\
u
\end{array}\right) \mid\left(\begin{array}{c}
\bar{x} \\
u
\end{array}\right) \in \mathcal{V}^{*}\right\} \\
& =\left\{\bar{x} \mid \bar{x} \in \mathcal{V}_{x}^{*}\right\}=\mathcal{V}_{x}^{*} .
\end{aligned}
$$

3. $\operatorname{ker} \Pi^{*}=\mathcal{W}_{x}^{*}$.

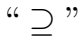

$$
\operatorname{ker} \Pi^{*}=\operatorname{ker} \Pi_{x} \Pi_{\mathrm{reg}} \Pi_{x}^{T} \supseteq \operatorname{ker} \Pi_{\mathrm{reg}} \Pi_{x}^{T}
$$

but

$$
\begin{aligned}
\operatorname{ker} \Pi_{\text {reg }} \Pi_{x}^{T} & =\left\{x \in \mathbb{R}^{n} \mid \Pi_{\text {reg }} \Pi_{x}^{T} x=0\right\} \\
& =\left\{x \in \mathbb{R}^{n} \mid \Pi_{\text {reg }}\left(\begin{array}{l}
x \\
0
\end{array}\right)=0\right\} \\
& =\left\{x \in \mathbb{R}^{n} \mid \Pi_{\text {reg }}\left(\begin{array}{l}
x \\
u
\end{array}\right)=0, \forall u \in \mathbb{R}^{m}\right\} \\
& \left.=\left\{x \in \mathbb{R}^{n} \mid \begin{array}{l}
x \\
u
\end{array}\right) \in \operatorname{ker} \Pi_{\text {reg }}, \forall u \in \mathbb{R}^{m}\right\} \\
& =\Pi_{x} \operatorname{ker} \Pi_{\text {reg }}=\Pi_{x} \mathcal{W}^{*}=\mathcal{W}_{x}^{*} .
\end{aligned}
$$

" $\subseteq$ "

choose $x \in \operatorname{ker} \Pi^{*}=\operatorname{ker} \Pi_{x} \Pi_{\mathrm{reg}} \Pi_{x}^{T}$, it results

$$
\begin{aligned}
& \Pi_{x} \Pi_{\mathrm{reg}} \Pi_{x}^{T} x=0 \Rightarrow \Pi_{x} \Pi_{\mathrm{reg}}\left(\begin{array}{l}
x \\
0
\end{array}\right)=0 \\
\Rightarrow & \Pi_{x} \Pi_{\mathrm{reg}}\left(\begin{array}{l}
x \\
u
\end{array}\right)=0, \forall u \in \mathbb{R}^{m} \\
\Rightarrow & \Pi_{x}\left(\begin{array}{l}
\bar{x} \\
u
\end{array}\right)=0 \Rightarrow \bar{x}=0
\end{aligned}
$$

where $\operatorname{col}(\bar{x}, \bar{u})=\operatorname{col}(0, \bar{u})=\Pi_{\text {reg }} \operatorname{col}(x, u)$. Now note that

$$
\left(\begin{array}{l}
0 \\
u
\end{array}\right) \in \operatorname{ker}\left[\begin{array}{ll}
I & 0 \\
0 & 0
\end{array}\right] \subseteq \mathcal{W}^{*}=\operatorname{ker} \Pi_{\text {reg }}
$$

therefore

$$
\begin{aligned}
& \Pi_{\text {reg }}\left(\begin{array}{l}
x \\
u
\end{array}\right) \in \operatorname{ker} \Pi_{\text {reg }}, \quad \forall u \in \mathbb{R}^{m} \\
\Rightarrow & \Pi_{\text {reg }} \Pi_{\text {reg }}\left(\begin{array}{l}
x \\
u
\end{array}\right)=0,
\end{aligned}
$$

and since $\Pi_{\text {reg }}$ is a projector

$$
\Pi_{\text {reg }}^{2}\left(\begin{array}{l}
x \\
u
\end{array}\right)=\Pi_{\text {reg }}\left(\begin{array}{l}
x \\
u
\end{array}\right)=0 .
$$

Finally for all $u \in \mathbb{R}^{m}, \operatorname{col}(x, u) \in \operatorname{ker} \Pi_{\text {reg }}$ and

$$
x \in \Pi_{x} \operatorname{ker} \Pi_{\mathrm{reg}}=\Pi_{x} \mathcal{W}^{*}=\mathcal{W}_{x}^{*}
$$

\section{B. Proof Theorem 5}

Let us apply the Wong sequences algorithm to the specific matrix pair in (7)

$$
\mathcal{V}_{i}=\left[\begin{array}{ll}
K & L \\
M & N
\end{array}\right]^{-1}\left(\left[\begin{array}{ll}
I & 0 \\
0 & 0
\end{array}\right] \mathcal{V}_{i-1}\right) .
$$

Considering now the definition of pre-image we obtain

$$
\begin{aligned}
\mathcal{V}_{i} & =\left\{\left(\begin{array}{l}
x \\
u
\end{array}\right) \in \mathbb{R}^{n+m} \mid\left[\begin{array}{ll}
K & L \\
M & N
\end{array}\right]\left(\begin{array}{l}
x \\
u
\end{array}\right) \in\left[\begin{array}{ll}
I & 0 \\
0 & 0
\end{array}\right] \mathcal{V}_{i-1}\right\} \\
& =\left\{\left(\begin{array}{l}
x \\
u
\end{array}\right) \mid K x+L u \in \Pi_{x} \mathcal{V}_{i-1}, M x+N u=0\right\}
\end{aligned}
$$

If we restrict this space to $x$ by defining $\mathcal{V}_{x}^{i}=\Pi_{x} \mathcal{V}_{i}$ we finally have

$$
\mathcal{V}_{x}^{i}=\left\{x \in \mathbb{R}^{n} \mid u \in \mathbb{R}^{m}, \begin{array}{c}
K x+L u \in \mathcal{V}_{x}^{i-1} \\
M x+N u=0
\end{array}\right\} .
$$

Similar calculations for $\mathcal{W}$ space sequence lead to

$$
\begin{gathered}
\mathcal{W}_{i}=\left[\begin{array}{ll}
I & 0 \\
0 & 0
\end{array}\right]^{-1}\left(\left[\begin{array}{ll}
K & L \\
M & N
\end{array}\right] \mathcal{W}_{i-1}\right) \\
=\left\{\left(\begin{array}{l}
x \\
u
\end{array}\right) \in \mathbb{R}^{n+m} \mid \exists\left(\begin{array}{c}
\bar{x} \\
\bar{u}
\end{array}\right) \in \mathcal{W}_{i-1},\right. \\
\left.\left[\begin{array}{ll}
I & 0 \\
0 & 0
\end{array}\right]\left(\begin{array}{l}
x \\
u
\end{array}\right)=\left[\begin{array}{ll}
K & L \\
M & N
\end{array}\right]\left(\begin{array}{l}
\bar{x} \\
\bar{u}
\end{array}\right)\right\} \\
=\left\{\left(\begin{array}{l}
x \\
u
\end{array}\right) \in \mathbb{R}^{n+m} \mid \exists\left(\begin{array}{l}
\bar{x} \\
\bar{u}
\end{array}\right) \in \mathcal{W}_{i-1},\right. \\
\quad x=K \bar{x}+L \bar{u}, 0=M \bar{x}+N \bar{u}\} .
\end{gathered}
$$

Define now the restriction $\mathcal{W}_{x}^{i}=\Pi_{x} \mathcal{W}_{i}$

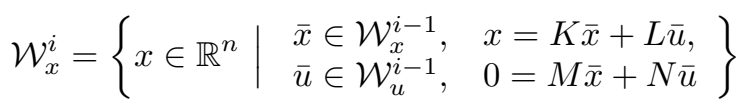

where $\mathcal{W}_{u}^{i}=\left[\begin{array}{ll}0 & I\end{array}\right] \mathcal{W}_{i}$ has the following property

$$
\mathcal{W}_{u}^{i}=\left\{\begin{array}{ll}
\{0\} & \text { if } i=0 \\
\mathbb{R}^{m} & \text { if } i>0
\end{array} .\right.
$$

\section{Moreover}

$$
\mathcal{W}_{x}^{1}=\Pi_{x} \mathcal{W}_{1}=\Pi_{x} \operatorname{ker}\left(\begin{array}{cc}
I & 0 \\
0 & 0
\end{array}\right)=\{0\}
$$

and thus, we can redefine the algorithm as it follows

$$
\mathcal{W}_{x}^{i}=\left\{\begin{array}{l|l}
x \in \mathbb{R}^{n} & \begin{array}{ll}
\bar{x} \in \mathcal{W}_{x}^{i-1}, & x=K \bar{x}+L \bar{u}, \\
\bar{u} \in \mathbb{R}^{m}, & 0=M \bar{x}+N \bar{u}
\end{array}
\end{array}\right\}
$$

with $\mathcal{W}_{x}^{0}=\{0\}$.

In such way it turns out that $\mathcal{W}_{x}^{i}=\Pi_{x} \mathcal{W}_{i+1}$. The two algorithms defining the $\mathcal{V}_{x}^{i}$ and $\mathcal{W}_{x}^{i}$ must converge after finitely many steps since they are defined to be just the projection of the full spaces $\mathcal{V}_{i}$ and $\mathcal{W}_{i}$ which in turn converge.

\section{REFERENCES}

[1] R. Frasca, M. Camlibel, I. Goknar, L. Iannelli, and F. Vasca, "Linear passive networks with ideal switches: Consistent initial conditions and state discontinuities," Circuits and Systems I: Regular Papers, IEEE Transactions on, vol. 57, no. 12, pp. $3138-3151$, dec. 2010.

[2] S. Trenn, "Switched differential algebraic equations," in Dynamics and Control of Switched Electronic Systems, ser. Advances in Industrial Control, F. Vasca and L. Iannelli, Eds. Springer London, 2012, pp. 189-216. [Online]. Available: http://dx.doi.org/10.1007/ 978-1-4471-2885-4_6

[3] M. K. Camlibel, W. P. M. H. Heemels, A. J. van der Schaft, and J. M Schumacher, "Switched networks and complementarity," Circuits and Systems I: Regular Papers, IEEE Transactions on, vol. 50, no. 8, pp. 1036-1046, aug. 2003.

[4] D. Liberzon, Switching in Systems and Control. Birkhauser Boston, 2003.

[5] K. Weierstrass, "Zur Theorie der bilinearen und quadratischen Formen," Berl. Monatsb., pp. 310-338, 1868.

[6] T. Berger, A. Ilchmann, and S. Trenn, "The quasi-weierstrass form for regular matrix pencils," Linear Algebra and its Applications, vol. 436, no. 10, pp. 4052 - 4069, 2012. [Online]. Available: http://www.sciencedirect.com/science/article/pii/S0024379509006776

[7] K.-T. Wong, "The eigenvalue problem $\lambda T x+S x$," J. Diff. Eqns., vol. 16 , pp. 270-280, 1974. 